\title{
De quoi et comment l'Europe rit-elle, au théâtre, de nos jours ?*
}

\section{Michel Corvin}

\section{(2) OpenEdition}

\section{Journals}

Édition électronique

URL : http://journals.openedition.org/recherchestravaux/155

DOI : 10.4000/recherchestravaux. 155

ISSN : 1969-6434

Éditeur

UGA Éditions/Université Grenoble Alpes

\section{Édition imprimée}

Date de publication : 15 octobre 2006

Pagination : 39-48

ISBN : 978-2-84310-099-4

ISSN : 0151-1874

\section{Référence électronique}

Michel Corvin, "De quoi et comment l'Europe rit-elle, au théâtre, de nos jours ?* », Recherches \& Travaux [En ligne], 69 | 2006, mis en ligne le 25 novembre 2008, consulté le 08 septembre 2020. URL http://journals.openedition.org/recherchestravaux/155; DOI : https://doi.org/10.4000/ recherchestravaux.155 


\section{De quoi et comment l'Europe rit-elle, au théâtre, de nos jours?*}

Depuis la fin du second conflit mondial, les occasions de rire, au théâtre, sont plutôt rares. La fin de la guerre a fait surgir des problèmes de conscience et les Allemands ont mis beaucoup de temps et noirci beaucoup de papier pour régler son compte au passé et du même coup régler les leurs. Les Espagnols et les Portugais sont encore plus mal lotis car les dictatures qu'ils ont subies ont été assez policières et méfiantes pour empêcher toute expression libre - et le rire vit de liberté - pendant des décennies. Quant aux pays d'obédience soviétique dont les régimes ont perduré jusqu'aux alentours de 1990, la censure s'y est exercée de façon parfois brutale, souvent sournoise, mais toujours assez efficace pour interdire toute autre forme d'art qu'un réalisme socialiste pétri de sérieux et de langue de bois. Et quand enfin, dans ces années 1990, on a pu croire l'Europe parvenue à un niveau de tranquillité démocratique propice à une créativité plus gratuite, les Balkans se sont enflammés et ce fut une succession d'horreurs et de massacres dont les États nouvellement constitués parviennent tout juste à sortir.

Si la situation politique, en de nombreux pays, n'est guère propice à la comédie et au comique durant la seconde moitié du XXe siècle, l'état de la société et les modes de vie des individus ne sont guère plus exaltants, qu'il s'agisse du dialogue avec soi-même ou avec l'autre (en couple ou non), avec la famille ou les relations professionnelles: toutes occasions de rencontre qui tournent le plus souvent à l'affrontement ou au dégoût. L'incapacité à échapper aux sollicitations dévastatrices de l'imaginaire, le déficit de lucidité face à soi, le lâcher-tout des pulsions, la sensation insidieuse d'un environnement hostile et

* Michel Corvin prépare une anthologie des textes de théâtre contemporains en Europe, à paraître aux Éditions Théâtrales.

Une première version de cet article a fait l'objet d'une communication lors du colloque «Pour un retour du rire aujourd'hui dans les institutions théâtrales» organisé le 22 mai 2006 au Théâtre des Célestins, à Lyon. 
instable, la perte des repères et, plus encore, la fin non assumée des interdits, tout cela favorise des déséquilibres psychiques propices à l'agressivité et à la violence, d'une bestialité inouïe, parfois. Comment en rire?

Et pourtant l'on rit. On va essayer de le montrer en parcourant la production théâtrale européenne des cinquante années qui séparent l'après-guerre de la fin du XX siècle, à l'exception de la France et des pays francophones, mieux connus et plus souvent étudiés.

Chose assez rare, il arrive qu'on cherche à provoquer un rire espiègle et gratuit. La Géorgienne Lasha Bughadzé a eu l'idée d'une salade historique plutôt cocasse: dans une sorte de pochade titrée Comédie française, elle met Marie-Antoinette en situation de parturiente accouchant en public, sur une scène, devant 2 ooo spectateurs ${ }^{\mathrm{I}}$. Comme elle souffre, elle s'écrie: "Que l'enfant meure. Que l'héritier meure! Vive la Révolution française!» Louis XVI hésite: "Élever l'héritier ou le tuer? N'auriez-vous pas pu me poser cette question il y a neuf mois?» À quoi la reine rétorque : "Vive la révolution de Juillet!», tandis que l'accoucheur nommé «Le Gynécologue de la tête aux pieds» l'encourage : "Majesté, poussez, poussez, je vous en prie!»

Se droguer est répréhensible, c'est bien connu, mais on peut se shooter à n'importe quoi, y compris à la littérature, ce qui, soit dit en catimini, compense la grisaille de la vie quotidienne en Russie, aujourd'hui. Tel est le thème développé par le jeune dramaturge Vladimir Sorokine dans Dostoüevski-trip. Le personnage Hi raconte un "voyage» qui a failli mal tourner, après avoir pris une dose de Tolstoï ${ }^{2}$ :

C'est quoi, je demande. De la bombe, il me dit. J'en prends. D'abord, rien de bien spécial. Un peu comme du Dickens, ou du Flaubert avec du Thackeray, et puis... bon... bon... vraiment du bon, un kif vraiment fort, large, une putain de puissance, mais alors après... après, vraiment l'horreur! L'horreur! (Il fait une grimace) Même Simone de Beauvoir m’a pas fait autant de mal que Tolstoï.

À l'exact opposé de cette attitude détachée - très caractéristique de la nouvelle génération russe -, le comique a été longtemps une arme, un moyen de survie même, sous les régimes policiers et absurdement bureaucratiques de l'Europe de l'Est. Que ce soit Havel en Tchécoslovaquie, Örkény en Hongrie ou Mrożek en Pologne, faire semblant d'épouser la logique de l'adversaire en la poussant à l'extrême, aller dans le sens de la doctrine officielle mais avec une telle surenchère que son ridicule éclate d'emblée, tel est l'un des procédés qui ont réjoui les spectateurs, quand la censure n'était pas assez vigilante

I. L. Bughadzé, Comédie française, Paris, L'Espace d'un instant, 2006.

2. V. Sorokine, Dostö̈evski-trip, Besançon, Les Solitaires intempestifs, $200 \mathrm{I}$. 
pour lire entre les lignes et étouffer toute représentation dans l'œuf. Dans Le rapport dont vous êtes l'objet de Vaclav $\mathrm{Havel}^{3}$, les palinodies serviles des petits chefs et leur bêtise hargneuse sur fond de chantage et de dénonciation calomnieuse sont percées à jour par un auteur dont on sait quel prix il a payé pour garder son esprit libre, et entier son droit de dire non. Et cela sans avoir l'air d'y toucher, en badinant autour d'une langue administrative évidemment incompréhensible, le pydétypède, dont les arcanes sont un jour portés aux nues et, un autre, jetés aux chiens.

István Örkeny, dans sa Famille Tot, est plus brutal et tout aussi absurdiste pour dénoncer (un peu à la façon de Ionesco dans Victime du devoir) les abus d'autorité des représentants du pouvoir ${ }^{4}$. Le Commandant s'est incrusté chez les Tot et s'acharne sur le seul membre qui lui résiste, le père de famille: au nom de la mystique stakhanoviste du travail, il lui interdit de s'arrêter de travailler, donc de manifester sa fatigue ou son ennui en bâillant: "Le bâillement, mon cher, dit le Commandant à Tot, ou plus exactement l'état d'âme qui provoque le bâillement, c'est aussi néfaste que la réflexion.» Dans son bataillon le Commandant a trouvé un moyen pour contrer ce travers: "Toutes les sentinelles ont un noyau de prune dans la bouche! Au moment de la relève, la garde descendante passe la consigne et le noyau de prune à la garde montante.» Chez les civils, faute de prunes, car la saison est passée, on cherche, on cherche le moyen d'empêcher Tot de bâiller; finalement on lui fourre une lampe de poche dans la bouche! On rit de lui et de sa lâcheté, car il accepte cette prothèse, mais bientôt on rira, d'un rire de revanche, voire de vengeance, quand Tot se rebellera et enverra promener son tortionnaire.

Le rire est la force des faibles, une façon de se dérober pour gagner du temps, une forme de dérision où le rire (désarmant) masque la part (agressive) de la satire. Dario Fo est le maitre de cette tactique, dans L'Enterrement du patron par exemple $e^{5}$ pour se venger, sans trop de risques, de leur patron, les ouvriers mettent en scène la cérémonie de son enterrement; tous rient - et nous avec eux car le spectacle est bien monté - sauf le commissaire de police qui tombe dans le panneau et se couvre de ridicule. Une des ouvrières, Franca, fait l'éloge funèbre du patron à coups d'antiphrases:

Bonnes gens, holà! bonnes gens, vous qui allez indifférents à votre travail, heureux de coopérer au bien commun des sociétés par actions! Arrêtez-vous un instant... et considérez l'injustice de la création... de l'univers: le soleil meurt mais renaît le lendemain; la lune aussi meurt, quand la pleine lune accouche, se divise et disparaît... mais c'est pour ressusciter. Pourquoi notre patron ne devrait-il pas,

3. V. Havel, Le Rapport dont vous êtes l'objet, Paris, Gallimard, 1992.

4. I. Örkény, La Famile Tot, Paris, Gallimard, 1968.

5. D. Fo, L'Enterrement du patron, dans Allons-y, on commence. Farces, Paris, Maspero, 1977 . 
ne pourrait-il pas ressusciter après sa mort, puisqu'il était pour nous le soleil et la lune tout ensemble... père, mère et sœur pour l'affection et les soins qu'il nous prodiguait... maîtresse et putain... pour tout le reste!?

Le rire que provoque Fo est partagé par les personnages et les spectateurs; c'est un rire de complicité peu différent de celui du personnage de Myriam dans la pièce du même nom écrite par le Russe Oleg Youriev où la malheureuse jeune femme, en pleine guerre, sur une ligne de front, voit sa masure envahie à tour de rôle par les représentants de trois factions armées, qui tous les trois, également lâches et avides, entendent la violer ${ }^{6}$. Rire compulsif de répétition, certes, mais il y a plus: Myriam ne s'en sort... qu'en disant oui - mais un oui de procrastination - et en recourant à quelques inventions dignes à la fois de la farce médiévale et des cocasseries à la Feydeau : elle est d'ailleurs la première à en rire.

\section{Détours satiriques}

Les ruses des petits qui mettent les rieurs de leur côté comportent une charge de satire - rire grinçant alors - très largement exploité sous toutes les latitudes sous la forme efficace et plaisante de la parabole. L'histoire racontée, le plus souvent marquée d'une touche d'insolite, se lit à un double niveau: niveau comique de la fable en tant que telle, niveau réflexif et sérieux des vrais enjeux de la pièce. On connaît bien le détachement élégant et cruel avec lequel la vieille dame de Friedrich Dürrenmatt explique froidement à ses concitoyens qu'elle consent à les couvrir d'or et de confort à condition qu'ils lui remettent en victime expiatoire un ancien amour de jeunesse qui l'avait plaquée après l'avoir engrossée 7 . Ce marchandage a la force de l'évidence: tout s'achète en ce monde. Autre approche très froide et très «suisse" dans Monsieur Bonhomme et les incendiaires du compatriote de Dürrenmatt, Max Frisch ${ }^{8}$. Le bourgeois Biedermann (Monsieur Bonhomme) est tellement sûr de lui et inconscient qu'il accueille à bras ouverts deux malfrats d'une jovialité suspecte; il les flatte et va même jusqu'à leur offrir les allumettes avec lesquelles ils incendieront la ville: parabole assez claire, mais mise en chansonnettes avec une fausse naïveté bon enfant, de l'urgence de l'action au moment précis - à saisir aux cheveux - où les terrorismes et autres fascismes peuvent être encore étouffés dans l'œuf.

Il s’agit de parabole encore dans la pièce de György Schwadja, Le Miracle. De Suisse nous passons en Hongrie, mais la fiction a pour même propos de faire rire en satirisant et, dans cette pièce, de susciter les réactions des spectateurs

6. O. Youriev, Myriam, dans Théatre russe contemporain, Arles, Actes Sud, I997.

7. F. Dürrenmatt, Le Retour de la vieille dame, Paris, Flammarion, 1957.

8. M. Frisch, Monsieur Bonhomme et les incendiaires, Paris, Gallimard, I96I. 
pour faire front aux abus et aux absurdités du «socialisme réel ${ }^{9}$ ». "Aveugle, tu vois!» telle se résumerait cette fiction où un ouvrier typographe presque aveugle, en fin de droits, n'entre plus dans aucune catégorie de patients pris en charge; en vertu de quoi il est déclaré en bonne santé et... renvoyé chez lui. On imagine la suite et comment l'aveugle se tenant pour guéri (puisqu'on le lui a dit!) va de catastrophe en catastrophe, plaisantes naturellement pour le spectateur qui, par son rire, manifeste sans doute son jugement de supériorité sur l'inconscience de l'aveugle, mais exerce surtout sa moquerie désabusée sur les délires de la bureaucratie «socialiste».

Des Soviétiques il est beaucoup question sous la plume des écrivains rebelles: l'agressivité se masque d'humour, sans que ce dernier émousse en rien la violence du refus. Joseph Brodsky est un Américain d'origine russe qui fut expulsé de son pays pour «décadentisme» et qui n'en obtint pas moins le prix Nobel en 1997. Le théâtre est son violon d'Ingres et sa pièce Démocratie est une charge savoureuse et précise contre le système soviétique, sa corruption, son hypocrisie et son cynisme ${ }^{\mathrm{IO}}$ : on y apprend comment on «fabrique» une démocratie (populaire) dans un des pays baltes. On y lit des phrases de cette eau: «Ce qui, avant la démocratie, était contre-révolution, devient passé glorieux avec la démocratie», ou: "Le triomphe de la justice prend exactement les mêmes formes que le triomphe de l'injustice. Autrement dit, il finit de la même façon: avec un gouvernement. » À cynisme, cynisme et demi, pourrait-on dire.

Chez Brodsky il a quelque chose de «dialectique»; chez Gregory Motton il est plus brutal dans une pièce comme Gengis parmi les Pygmées. Deux espèces de clowns, Gengis et Tata jouent à mimer les méfaits de la société d'exploitation; Gengis se déguise en Philippine et se rend avec Tata à son lieu de travail ${ }^{11}$ :

GENGIS: C'est quoi cette horrible odeur?

TATA: C'est une rivière d'eaux usées mélangées avec un délicieux produit d'entretien associé à un désodorisant d'atmosphère. C'est grave propre han ?

GENGIS : Indubitablement. Je suis content de voir que tu prends encore soin de l'environnement. (Il respire profondément. Bavardant négligemment alors quills se rendent à ses quartiers) C'est super. Je ne jure que par ça. Le truc super avec un bon désodorisant d'atmosphère c'est qu'en cas de réelle urgence tu peux carrément te le fourrer dans le cul.

TATA: Bon, voilà ton boudoir.

GENGIS: Comment oses-tu! ça, un boudoir? Ce n'est rien que des traînées de crasse par terre. Et il y a des dizaines d'autres filles.

TATA: Je crains que ce soit ainsi que vivent les Philippins.

GENGIS: C’est répugnant.

9. G. Schwadja, Le Miracle, Paris, Éditions théâtrales, 1996.

Io. J. Brodsky, Démocratie, Die, Éditions A. Dié, 2003.

II. G. Motton, Gengis parmi les Pygmées, Paris, Éditions théâtrales, 2004. 
TATA: Bon, je dois inspecter ta culotte régulièrement pour vérifier que tu as tes règles et détecter le moindre signe d'excitation sexuelle, ce qui n'est pas permis. GENGIS: Quel dommage. Pourquoi pas?

TATA: On ne peut pas employer de femmes enceintes, elles prennent trop de place, elles pètent trop souvent et ensuite elles veulent du temps libre pour accoucher. GENGIS: Je promets que si jamais j'accouche je le mettrai directement dans les cabinets.

Motton ne fait pas dans la dentelle et sa vulgarité délibérée a un effet de repoussoir un peu comme les ressassements infinis auxquels Thomas Bernhard a consacré l'essentiel de sa production dramatique: le monde entier et plus particulièrement l'Autriche, quelle que soit la classe sociale qu'il épingle, sont remplis d'imbéciles et de lâches, de nazis en somme ${ }^{\mathrm{I} 2}$ :

[...] ces cinquante dernières années ceux qui / nous gouvernent ont / tout détruit / et ce n'est plus réparable / les architectes ont tout détruit / par leur stupidité/ les intellectuels ont tout détruit / par leur stupidité / le peuple a tout détruit / par sa stupidité / les partis et l'Église / ont tout détruit par leur stupidité / qui a toujours été une stupidité pleine de / bassesse / et la stupidité autrichienne est une stupi / dité de bout en bout repoussante / L'industrie et l'Église / sont responsables du malheur autrichien / L'Église et l'industrie ont depuis toujours / été responsables du malheur autrichien / les gouvernements sont totalement dépen / dants / de l'industrie et de l'Église / il en a toujours été ainsi / et c'est en Autriche que tout a toujours été le pire / tous ont toujours couru après la stupidité / l'esprit a toujours été piétiné / L'industrie et le clergé tirent les ficelles du mal autrichien [...] (Place des héros)

Il ne faudrait pas croire que l'Autriche soit le seul objet de sa vindicte: il faut lire Le Faiseur de théâtre pour voir jusqu'à quel degré de misogynie spasmodique - à moins que ce ne soit de l'humour au énième degré - peut parvenir un écrivain que l'on sait passionné mais que l'on croit sensér ${ }^{13}$. Dira-t-on avec Voltaire: "Ce qui est excessif est insignifiant» et se contentera-t-on de rire, mais de Bernhard? Je pense plutôt que la surenchère forcenée de l'écrivain, outre son effet comique (comique traditionnel de répétition, d'entassement et de structure en boucle) a un effet purgatif: son irrévérence est un salutaire sursaut contre la langue de bois et les consensus mous.

Pour élever une voix discordante dans le concert européen, on peut recourir à une technique moins outrée mais tout aussi ironique. Le Lituanien Marius Ivaskevicius s'y emploie dans sa pièce Le Voisin; il s'y souvient des coq-à-l'âne et autres enchaînements illogiques de Ionesco pour célébrer «l'amitié» entre les peuples ${ }^{\mathrm{I}}$ : "Je ne pense pas, dit Vaclov, que tous les Biélorusses soient des voleurs. Vous savez, tout comme les Juifs, eux non plus on ne peut pas les

I2. T. Bernhard, Place des héros, Paris, L'Arche, I990.

13. Id., Le Faiseur de théâtre, Paris, L'Arche, 1997.

I4. M. Ivaskevicius, Le Voisin, Presses universitaires de Caen, 2003. 
distinguer des Russes. Il y a en a, bien sûr, quelques-uns qui volent, d'autres boivent ou trompent leur femme. Rarement ils se battent. Quelques-uns seulement, peut-être. Les Biélorusses, eux, en général, aiment montrer la direction à prendre. Ils ne sont jamais pressés, et ça c'est déjà bien.» Quand on songe que c'est un Russe de Lituanie qui parle; quand on sait en quelle estime ce pays satellisé tenait son occupant russe, ces quelques phrases contiennent leur pesant de satire, surtout perceptible pour les spectateurs du cru.

On a alors affaire à du comique pour initiés, relativement inexportable. C'est aussi le cas du rire d'autodérision dans lequel les Juifs sont passés maîtres. George Tabori en sème à foison les exemples notamment dans Les Variations Goldberg ${ }^{15}$. À la scène 9 "la scène est vide à l'exception d'une grande échelle au sommet de laquelle est accrochée une pancarte "Sinaï" ainsi qu'un buisson». Les Hell's Angels font leur entrée et chantent:

LE PREMIER DES HELL'S ANGELS :

Nous somm's les méchants Juifs

Comm' vous les connaissez

Avec leurs affreux pifs

Gauchistes et drogués

LE DEUXIÈME :

On fait un' sacrée bande

De bêtes sexuelles.

On baise mêm' des All'mandes

$\mathrm{Y}$ en a parfois de belles

Mais maintenant qu'c'est légal

Elles nous sont bien égales. [...]

Et une fois que les Hell's Angels ont fini de chanter, arrive, accompagné du Veau d'or, M. Jay, le metteur en scène, qui proclame:

Bafouez toutes ces lois qui vous emmerdent: mentez, volez, tuez, baisez comme ça vous chante, adorez d'autres dieux, convoitez la femme de votre prochain, et surtout dansez autour du Veau d'Or, comme si c'était sur le mont de Vénus. Et si le buisson se met à flamber, compissez-le.

On rit, sans doute, mais un peu jaune: la provocation n'aurait-elle plus de limites?

\section{Fantaisies comiques}

À partir du moment où l'on met le doigt dans l'engrenage de la transgression, toutes les fantaisies, les plus agressives comme les plus anodines, deviennent

I5. G. Tabori, Les Variations Goldberg, Paris, Éditions théâtrales, I997. 
possibles. C'est le Bulgare Raditchkov qui dans Janvier fait cohabiter les humains avec de curieux fantômes bienveillants qui brouillent aimablement les limites entre réel et surréel ${ }^{16}$; c'est le Suisse Köbeli qui dans Peep-show dans les Alpes transforme la vie paysanne des montagnes en spectacle folklorique parfaitement mis au point à l'usage de touristes voyeurs ${ }^{17}$; c'est le Russe Slavkine qui dans Le Tableau lance un peintre malheureux sur la piste de son tableau avec lequel il s'identifie ${ }^{18}$; c'est encore la Britannique Churchill qui, dans Top Girls, raconte l'aventure désastreuse et désopilante de Jeanne la Papesse, que seule sa grossesse finira par dénoncer ${ }^{19}$. "Je t'aime, je te tue " est un aphorisme que l'on voit rarement mis en œuvre en dehors de la tragédie classique et, alors, de façon fort sérieuse. L'Allemand Franz Xaver Kroetz dans Une affaire d'homme réussit à transformer un massacre "amoureux" en partie de plaisir (pour le spectateur) ${ }^{20}$. Otto et Martha ou un fusil pour deux. Par émulation de courage, ils se tirent dessus avec l'arme qu'ils se passent loyalement l'un à l'autre. La fin de la pièce est un chef-d'œuvre d'humour macabre:

OTTO: Prends ton temps. Je me sauve pas. Il lui amène le fusil. Je retourne à ma place.

MARTHA: Juste, il faut une règle en toute chose. Elle tire et l'atteint.

Otto chancelle contre le mur.

Martha dépose le fusil au sol et le pousse du pied. Prends-le.

OTTO: Merci.

Ils échangent encore chacun un coup de fusil, puis Martha tombe en avant sur le visage et reste couchée sans bouger. Otto regarde, repose le fusil, s'approche de Martha.

Tu te rends?

Si l'on fait abstraction, pour tous ces derniers textes, de leur force critique pour ne retenir que leur puissance d'invention, on fait un pas vers de nouvelles formes de comique et de comédie qui pourraient être nommées comédies du langage ou comique intellectuel. L'Autrichien Werner Schwab a su, pour camper ses personnages dérisoires happés par le non-sens, leur faire parler un étrange sabir qui, même en traduction, nous donne l'impression de côtoyer des êtres d'un autre monde ${ }^{21}$. Dans Escalades, le héros se nomme Helmut Combustion et dans un mouvement d'autodestruction il demande au vendeur de saucisses d'entasser toujours davantage de moutarde forte sur lui, tandis que le représentant de l'ordre bourgeois, le «Cadre de la Caisse d'épargne", le morigène:

I6. Y. Raditchkov, Janvier, Paris, Éditions théâtrales, 2002.

17. M. Köbeli, Peep-show dans les Alpes, Paris, Éditions théâtrales, I995.

I8. V. Slavkine, Le Tableau, Arles, Actes Sud, 1992.

19. C. Churchill, Top Girls, Paris, L'Arche, 1997.

20. F. X. Kroetz, Travail à domicile suivi de Une affaire d'homme, Paris, L'Arche, 1997.

2I. W. Schwab, Escalade ordinaire suivi de Extermination du peuple, Paris, L'Arche, 1998. 
Au travail. / Le travail appelle comme la forêt. / Le travail est la plus unique chose prophylactique / comme n'importe quel maléfice. / Une personne active sexuellement majeure est l'imago de la population efficaciquement active. / Une personne sans travail est le phimosis de la nature humaine pleinement valable.

La force comique de ce langage, en constant décalage, est peut-être trompeuse ou du moins ne doit pas dissimuler les intentions politiques de Schwab: ses malheureux pantins, souvent bêtes et méchants, sont des marginaux de la société de consommation, incapables d'en tirer profit mais capables d'en juger les méfaits, même s'ils en parlent mal le langage. En jetant sa saucisse, Helmut, "combustion indéfinissable», la définit comme "nourriture pour cochons à placenta postnatal saucissaire»!

Il y a beau temps que le théâtre joue avec lui-même et en tire des effets comiques de dédoublements et de reflets dans son propre miroir : qu'on pense à L'Impromptu de Versailles ou à L'Illusion comique. Les auteurs contemporains restent dans la même lignée même s'ils en renouvellent les procédés : un Tom Stoppard dans Rosencrantz et Guildenstern sont morts, inverse les données fabulaires de Hamlet et fait des seconds couteaux que sont les deux anciens amis du prince de Danemark les héros malgré eux d'une aventure tragique à laquelle ils s'efforcent vainement d'échapper ${ }^{22}$ : comme ils sont déjà "écrits" par Shakespeare, ils n'ont aucune chance de s'en sortir. Le spectateur, lui, se sait protégé des coups de ce destin-là et il rit de leur impuissance; il y a une bonne part de méchanceté dans certains rires!

Le Roumain Visniec, l'Allemand Schimmelpfennig, le Britannique Ayckbourn, voire l'Italien De Filippo, jouent plus classiquement, mais avec maestria, avec les espaces du dedans/dehors, avec les empiétements du fictif sur le réel, avec les surimpressions des temps, en somme avec toutes les conventions théâtrales qui nous font prendre naïvement les personnages pour des personnes, les paroles dites pour l'expression vraie de sentiments éprouvés, l'histoire racontée pour un morceau d'existence. Tout est faux au théâtre, certes. Aujourd'hui on surenchérit sur ses trucages, y compris dans le sens de l'humour macabre comme dans L'Enfant froid, parodie d'une pièce de boulevard écrite par l'Allemand Marius von Mayenburg ${ }^{23}$. Le spectateur est toujours friand d'assister au démontage des procédures de fictionalisation; il se sent un peu démiurge, complice et confident de l'auteur; heureux même - car le comique produit aussi comme une sensation de bonheur - de se sentir intelligent à être d'intelligence avec le dramaturge.

22. T. Stoppard, Rosencrantz et Guildenstern sont morts, Paris, Seuil, 1967.

23. M. von Mayenburg, Eldorado suivi de L'Enfant froid, Paris, L’Arche, 2004. 
On a souligné à maintes reprises que des mécanismes psychologiques et intellectuels sont à l'origine du comique et par voie de conséquence des procédés qui déclenchent le rire. Il ne faudrait pas pour autant oublier que le rire sait être libre et gratuit quand il est une manifestation, sans raison particulière, de la joie de vivre. Cette veine-là, les Français l'exploitent peu, sauf chez des «irréguliers» comme les auteurs de fatrasies du moyen âge, Rabelais ou Jarry, car en en faisant une arme et un contre-pouvoir, les écrivains français ont rationalisé et domestiqué le rire. Ce qui n’est pas le cas du Bulgare Boytchev pour qui le rire est une source de rêve. Dans Cette chose-là les petites gens qui font la pièce se laissent emporter dans une eschatologie burlesque où ils brossent en souriant le destin, plutôt morose et terne, des générations futures ${ }^{24}$ : cette chose-là, indicible et innommée, que l'on essaie de cerner et qui donnerait un sens à la vie, on ne peut guère l'amadouer qu'en se mettant à chanter. "Qu' estce qu'on va chanter?» demande Prodan, et Dinko répond: «Je ne sais pas. Ce qui nous vient de l'intérieur. " Un rire dans l'âme pour ainsi dire.

Dans Bailegangaire de l'Irlandais Thomas Murphy, la vieille Momo raconte la saga funèbre de toute sa maisonnée, scandant d'un rire spasmodique chaque rappel d'une mort, d'une catastrophe ou d'une bagarre ${ }^{25}$. Comme le signale l'indication scénique: "les "bih hih hih" qui ponctuent son histoire sonnent plutôt comme des pleurs... des pleurs retenus... que comme des rires». Et Momo tire de ses rires une philosophie de l'existence: philosophie de résistance où le rire est un antidote contre la misère et la mort:

Les histoires n'en terminaient pas d'arriver et les volées et les hourras! Tous les présents là, la tête renversée en arrière, s'abandonnant à des festivités de grands rires; les misérables et les abandonnés, calamiteux et perdus, les oubliés et tourmentés, les esseulés et les désespérés, en guenilles et crasseux, appauvris, misérables, émaciés et maladifs, les yeux grands comme des soucoupes, ridiculisant et défiant leur lot sur cette terre... étincelants de leur défi... leur défi et leur rejet, appelant de leurs vœux ce qui risquait de leur tomber dessus ou se soucierait de le faire... lançant des vociférations de refus au ciel par-delà le plafond! oh! oh! ock kkk ah ah!... Nuit fastueuse entre toutes.

Nuit fastueuse faite de rien d'autre que d'une bolée d'air vital qui s'exprime en souffle rieur.

24. H. Boytchev, Cette chose-là suivi de L'Homme souterrain, Paris, L'Espace d'un instant, 2006.

25. T. Murphy, Bailegangaire, Paris, Éditions théâtrales, 1993. 